\title{
Do science blogs promote reader engagement? An experimental comparison between a blog post and traditional expository text
}

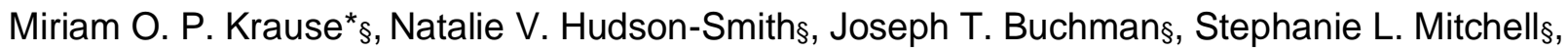

[PREPRINT - Please do not cite without authors' consent]

$\S$ Department of Chemistry, University of Minnesota, 207 Pleasant St SE, Minneapolis, Minnesota 55455, United States

\section{Corresponding Author}

${ }^{*}$ Dr. Miriam O. P. Krause, University of Minnesota and Center for Sustainable Nanotechnology, mkrause@umn.edu

\section{Acknowledgements}

This work was supported by the National Science Foundation under the Center for Sustainable Nanotechnology, CHE-1503408. The CSN is part of the Centers for Chemical Innovation Program. J.T.B. and N.V.H.-S. acknowledge support from a National Science Foundation Graduate Research Fellowship (grant number 00039202). S.L.M. acknowledges support through a NIH Chemistry-Biology Interface Training Grant 5T32GM008700-18 as well as the Doctoral Dissertation Fellowship from the University of Minnesota. We are grateful to the University of Minnesota Driven to Discover Building for providing space to collect data, and to the Minnesota State Fair attendees for filling out our survey. The authors are thankful to Emily Kurtz for her extensive statistical analyses and discussion of the collected data. The authors are grateful to Dr. Amanda A. Olsen for her work generating the maps that show geographical distribution of the MN participants. We also acknowledge Dr. Emily Tollefson, Dr. Sunipa Pramanik, and Linus Pomerantz for their help with data collection. 


\section{Abstract}

Science blogging is a common practice for communication with broad audiences; however, the effectiveness of blogs for promoting public engagement with scientific material has not been fully assessed. This study investigated reader engagement after reading either a scientific blog post or a related expository-style article by comparing whether participants volunteered for further email communications at the end of the study. We found that the blog group showed significantly greater engagement than the expository reading group. Results provide evidence for the effectiveness of blogging as a science outreach tool, and suggest potential future research topics related to age, gender, and geography.

\section{Introduction}

Blogs have been a popular platform for science outreach since the mid-1990s (Zivkovic, 2012), and continue to be prolific despite the rise in social media, video, and other avenues of informal science communication (Jarreau \& Porter, 2018). By one estimate, $24 \%$ of scientists have blogged about their research or some other science in the past (Jarreau, 2015). Scientists blog on personal blog platforms (e.g. Small Pond Science (McGlynn, 2019), The Culture of Chemistry (Francl-Donnay, 2019)) as well as blogging networks (e.g. Chemistry Blog (Chemistry Blog, 2019), Scientopia (Scientopia, 2019)) and blogs run by scientific outlets (e.g. Scientific American (Scientific American, 2019), Nature.com Blogs (Nature, 2019), AGU Blogosphere (American Geophysical Union, 2019)). Scientific blogging and bloggers may have goals of communicating among scientists (e.g., (McGlynn, 2019), (Natelson, 2019)) and/or communicating to a non-scientist public (e.g. Sustainable Nano (Center for Sustainable Nanotechnology, 2019), Bad Astronomy (Plait, 2019), ScienceGrrl (ScienceGrrl, 2019). For communicating to a nonscientist public, blogging provides various advantages to the scientist communicator: online communication is highly accessible, requires few resources (other than time), and shows some evidence of being effective at improving knowledge and disseminating research findings (Su, Cacciatore, Scheufele, Brossard, \& Xenos, 2014), (Su, Akin, Brossard, Scheufele, \& Xenos, 2015), (Hoang, McCall, Dixon, Fitzgerald, \& Gaillard, 2015). It can also provide opportunities for two-way communication between the scientist and their non-scientist audience (Collins, Shiffman, \& Rock, 2016). Despite the proliferation of science blog content, systematic research into whether blogs accomplish the science communication goals for which they are designed, particularly related to audience engagement, is still emerging. The definition of engagement varies among practitioners and researchers (Lewenstein, 2016), but may comprise multiple factors including aspects of learning, positive attitude, and enjoyment.

The reasons for limited research into science blogging effectiveness may be related to the very flexibility that make blogs so appealing as a medium, i.e. bloggers can tailor content to their own preferences and interests (Jarreau, 2015), and readers can access the content at any time, from any location, with any degree of regularity, and without direct interaction with communicators. The great variability and flexibility in goals, tactics, audiences, and styles can make it challenging to conduct systematic investigations about whether blogs in general are "effective." In a 2010 study of eleven example blogs, Kouper went as far as to say that "science blogs are too heterogeneous to be understood as an emerging genre of science communication" (Kouper, 2010). 
The current study side-steps the variability problem by focusing on a specific blog, Sustainable Nano (sustainable-nano.com, (Center for Sustainable Nanotechnology, 2019), and a specific goal related to reader engagement. The Sustainable Nano blog is a collaborative outreach blog produced by the Center for Sustainable Nanotechnology (CSN), a multi-institute Center for Chemical Innovation funded by the U.S. National Science Foundation. Sustainable Nano posts are mostly written by graduate students and faculty in the CSN, which provides a professional development opportunity in informal science communication for writers, as well as facilitating consistent publication on a variety of topics by distributing the responsibility for writing across a large number of authors. Outreach goals for the blog are to increase public understanding of - and engagement with - scientific concepts related to nanotechnology and sustainability. In their recent literature review on the challenges of communicating with the public about nanotechnology, Boholm and Larsson argue that it is important for nanoscientists to engage actively with the public and increase transparency to expand public understanding and acceptance of new technologies (Boholm \& Larsson, 2019). Despite its prevalence in commercial and medical applications, nanotechnology is still somewhat unfamiliar to the general public, and research has shown that public perceptions of nanotechnology can vary widely depending on factors such as media exposure, education, and attitude toward environmental risks. Relaying concepts related to nanotechnology is difficult because the unique properties and scale of nanoparticles can seem inaccessible to non-scientists, and amongst the public there are generally low perceived risk and uncertainty about the benefits. Considering these factors led Boholm and Larsson to conclude that "nanotechnology is generally not an issue that spurs public engagement."

Scheufele and Lewenstein (Scheufele \& Lewenstein, 2005) used the "low-information rationality" model to explain this engagement challenge: if public consumers both perceive little threat from nanotechnology and assume that it would take a significant effort to increase their understanding, they are unlikely to consider it worthwhile to pursue additional information. This suggests that the Sustainable Nano blog, with its aim to present information that is easily accessible (i.e. easy to find with a targeted web search and requiring little effort to increase understanding) is well-positioned to increase audience engagement. The purpose of the present study was to directly measure whether exposure to a Sustainable Nano blog post increased reader engagement relative to a Wikipedia article of comparable length. For the purposes of our study, we chose to measure "engagement" behaviorally, based on whether participants signed up to receive future emails related to science blogs after participating in the study. This simple metric could indicate any combination of learning, positive attitude, enjoyment or other factors. We hypothesized that exposure to a single Sustainable Nano blog post would not lead to improvements on a knowledge quiz about nanoscience (since the concepts on the quiz were not explicitly addressed in the sample post); rather, we predicted that participants who were randomly assigned to read the blog post would be more likely to sign up for further communications than those assigned to the Wikipedia reading group. Knowing whether exposure to the blog leads to any further information-seeking engagement is useful since, like many other bloggers (Jarreau, 2015), we post blogs in the hope that our efforts will be appealing to non-scientists and increase their interest in science. 


\section{Methods}

\section{Recruitment and Participants}

All participants were recruited at the Driven to Discover building (and through D2D web or app information) at the 2017 Minnesota State Fair. D2D is a University of Minnesota-sponsored program for connecting researchers and members of the public for study participation (http://d2d.umn.edu). Potential participants self-identified by coming to the D2D building at the State Fair and approaching the booth for this study, where research personnel could then give them more information. In order to participate in the study, participants had to be at least 18 years old and self-identify as fluent readers of English. Due to the ready availability of alcohol at the State Fair, D2D studies are encouraged to include a specific exclusion criterion about intoxication: in this case, participants who researchers judged to be intoxicated or who self-reported having had three or more alcoholic drinks in the past hour were excluded. Screening questions about age, reading fluency, and alcohol consumption were included as part of the consent process. Incentive to participate in the study was the opportunity to enter in to a drawing to win one of ten $\$ 10$ Starbucks gift cards. All procedures were approved by the University of Minnesota Institutional Review Board.

\section{Design}

The study used a between-subjects design, employing a survey structure to assess participants' engagement (interest in receiving more information) after reading either a Sustainable Nano blog post or a control text of similar length. The survey also included measures of knowledge and attitudes about nanotechnology and science in general both before and after the readings.

After the consent process, all participants completed the same 4-part procedure:

Part 1: Pre-reading demographics, attitude, and knowledge measures. Participants provided demographic information such as age, race, education, occupation, and frequency of reading science blogs. They then answered a knowledge assessment about nanotechnology based on the five-question quiz from Su et al. (Su et al., 2014). Finally, participants completed Likert-like ratings about their attitudes toward science (e.g. "strongly agree" to "strongly disagree" ratings for questions such as "Science classes are a waste of time" or "I would enjoy visiting a science museum on the weekend.") which were modified from a the Test of Science-Related Attitudes (TOSRA) (Fraser, 1981). 
Table 1. Pre-reading knowledge \& attitude questions

Part 1. What do you know about nanotechnology?

We are interested in what you already know about the area of science called nanotechnology. Answer each question as "definitely true," "likely true," "likely false," "definitely false," or "don't know." [The survey displayed questions on a grid so participants could easily select from 1-5 (definitely true to definitely false or don't know) for each question.]

1. Nanotechnology involves materials that are not visible to the naked eye.

2. A nanometer is a billionth of a meter.

3. Experts consider nanotechnology to be the next industrial revolution of the U.S. economy.

4. So far, there are only a few dozen consumer end products on the market using nanotechnology.

5. Nanotechnology allows scientists to arrange molecules in ways that do not normally occur in nature.

Part 2. What do you think about science?

We are interested in your overall opinions about science. There are no correct or incorrect answers in this section. Answer each question as "strongly agree," "agree," "not sure," "disagree," or "strongly disagree." [The survey displayed questions on a grid so participants could easily select from 1-5 (strongly agree to strongly disagree) for each question.]

1. Money spent on science is well worth spending

2. Science is humanity's worst enemy

3. I get bored when watching or listening to science programs

4. Finding out new things is important

5. Scientists are less friendly than other people

6. Science classes are a waste of time

7. I would enjoy visiting a science museum on the weekend

8. Science can help to make the world a better place in the future

Part 2: Reading. Participants in the experimental (blog) group read a Sustainable Nano blog post, "Mimicking Nature's Nanotechnology: From a Butterfly Wing to Anti-Counterfeit Technologies" (Doğangün, 2015). Participants in the control (Wiki) group read a text of similar length $(\sim 1,000$ words) from the Wikipedia entry on butterflies (Wikipedia, 2017). Both "articles" included text and images and were formatted simply, without any logos or website-specific formatting. After this first reading, both groups were given the option to read a second selection, the Sustainable Nano post "What Do Gold Nanoparticles Have to Do With Wrinkly Skin?" (McGeachy, 2014). (See Supplemental Information for all three complete readings.) Electing to read a second article was initially conceived as an additional measure of engagement, though this did not come to fruition, as discussed further below.

Part 3: Post-reading attitude and knowledge measures. After the reading section, all participants were presented with their pre-reading responses to knowledge and attitude measures and were given the opportunity to change any responses they wanted before concluding the study.

Part 4: Engagement measures and conclusion. On the final page of the study, all participants were thanked and then received the opportunity to sign up for three follow-up items: (1) to receive an email list of recommended science blogs, (2) to receive email updates from the Sustainable 
Nano blog, and (3) to enter a prize drawing for a $\$ 10$ gift card. Participants who signed up for follow-up emails received them, and ten prizes were awarded randomly.

Filling out items 1 and 2 comprised our dependent variables measuring "engagement." Selecting to read a second article during Part 2 of the study was originally envisioned as an additional measure of engagement; however, as explained further in the Results and Discussion section, we found that, regardless of random group assignment, people who chose to read the second article were significantly more likely to sign up for the follow-up engagement activities than those who read only one article. Because of this, participants who read the second article were excluded from the final blog vs. Wiki group comparisons in an attempt to isolate the effects of reading group from any predisposition to demonstrate engagement with science texts.

\section{Data Analysis}

Comparisons of the different groups were completed using $\mathrm{R}$. To determine statistical significance, $x^{2}$ tests were run for most comparisons, and Student's t-test was used to compare engagement for the blog vs Wiki single article readers.

\section{Results and Discussion}

\section{Recruited participants}

A total of 232 participants were recruited for the study. Four were excluded because they did not complete the survey past the consent page, leaving 228. Demographics for all participants who completed the survey are provided in the Supplemental Information.

\section{Emergent "pre-engaged" subgroup}

As described in the Methods section, we originally conceived of three measures of reader engagement and hypothesized that they would collectively show differences between participants who read the blog post vs. the Wikipedia article: (1) choosing to read a second article, (2) signing up to receive a one-time email recommendation list of science blogs, and (3) signing up to receive email notifications from the Sustainable Nano blog. However, in the course of our preliminary analysis, we discovered that not only did blog vs. Wiki group not predict whether participants would choose to read a second article, but the data regarding email signups from the "2nd article" subgroup overwhelmed any potential overall effect of group assignment. That is, regardless of group assignment (blog vs. Wiki), people who chose to read a second article were significantly more likely to also demonstrate one or both of the other engagement behaviors compared to those who did not read the second article, $X_{2}^{2}(2, N=228)=23.037, p=9.95 \times 10-6$ (Figure 1).

This unexpected pattern led us to suspect that, rather than measuring engagement due to a participant's first assigned reading (blog vs. Wiki) in our study, selection of the second reading was an indication of their general pre-existing engagement. In other words, some participants came to our study already inclined to seek out science blogs and similar content, whether they were assigned to the blog or Wiki condition of our particular study.1 This is not entirely surprising,

1 FOOTNOTE: Our survey was designed to provide another way to inform this assumption, by asking "How often do you read blogs about scientific issues or emerging technologies?" [ $1=$ never to $5=$ sometimes to $10=$ very often]. Although the "pre-engaged" subgroup may not have all been blog readers, we would predict that those who are active readers of science blogs would be more likely to be part of the 
given that participants had self-selected to enter the Driven to Discover research building and volunteered to participate in a study called "Science Survey." Because of the emergence of this "pre-engaged" subgroup of participants, we decided to examine our primary hypothesis (that reading the blog post would be more conducive to engagement behavior than reading the Wiki post) by using data only from participants who did not elect to read a second article.

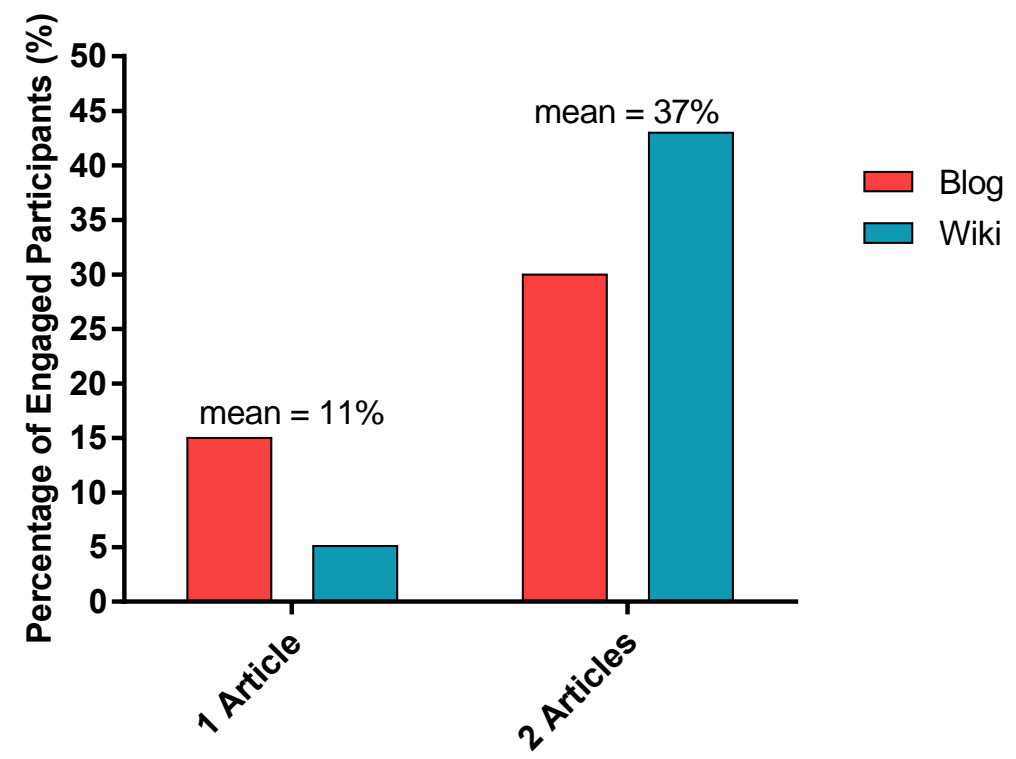

Figure 1. The percentage of participants who signed up for one or both email follow-ups (engagement) for each sub-group (1 article vs. 2 article readers) demonstrates that, regardless of random group assignment, participants who chose to read a second article were more engaged than the participants who only read a single article. $X_{2}(2, N=228)=23.037, p=9.95 \times 10-6$.

After eliminating the two-article ("pre-engaged") subgroup, 130 participants remained (71 in the blog group and 59 in the Wiki group). Their demographic characteristics, as shown in Table 2, remained similar to the balanced distribution of the initial recruited population (see supplementary information), except that the blog group had a disproportionately high number of women (65\% compared to $51 \%$ in the Wiki group). A chi-square analysis showed that gender and engagement were independent of each other $\left(x^{2}(1, N=130)=0.033, p=0.86\right)$, so we believe it unlikely that blog vs. Wiki group differences were driven by different gender proportions.

Table 2. Demographic information of the single-article subgroup, after excluding participants who did not elect to read a second article ("pre-engaged" subgroup).

"pre-engaged" subgroup. Unfortunately, due to an error in our survey flow logic programming, responses to that question were not saved by the software and were therefore not available for analysis. 


\begin{tabular}{|l|l|l|}
\hline & \multicolumn{2}{|l|}{ Single-article subgroup $(\mathrm{n}=130)$} \\
\hline & Blog $(\mathbf{n}=\mathbf{7 1})$ & Wiki $(\mathbf{n}=59)$ \\
\hline Age (mean \pm SD) & $44.9 \pm 15.6$ & $46.9 \pm 16.1$ \\
\hline Gender (M:F:NR) & $25: 46: 0$ & $29: 30: 0$ \\
\hline $\begin{array}{l}\text { Education } \\
\%<B A\end{array}$ & 21.1 & 22.0 \\
$\%$ BA & 33.8 & 37.3 \\
\hline Grad & 45.1 & 40.7 \\
\hline Geography (\% urban) ${ }^{*}$ & 72.3 & 77.8 \\
\hline Race (\% white) & 94.3 & 86.4 \\
\hline
\end{tabular}

$\mathrm{SD}=$ standard deviation, $\mathrm{M}=$ male, $\mathrm{F}=$ female, $\mathrm{NR}=$ no response, $\mathrm{BA}=$ bachelor's degree, Grad = graduate degree

*Because we only coded Minnesota zip codes as urban/non-urban, this percentage excludes the 8 participants from outside of Minnesota.

\section{Blog vs. Wiki groups}

Our primary hypothesis was that participants randomly assigned to the blog group would demonstrate greater engagement (i.e. signing up for one or both of the follow-up email opportunities), than those assigned to the Wiki group. This hypothesis was supported, as shown in Figure 2: with $15.5 \%$ of participants demonstrating engagement, the blog group was significantly more engaged than the Wiki group $(5.1 \%), t(117.96)=2.00, p=0.048$.

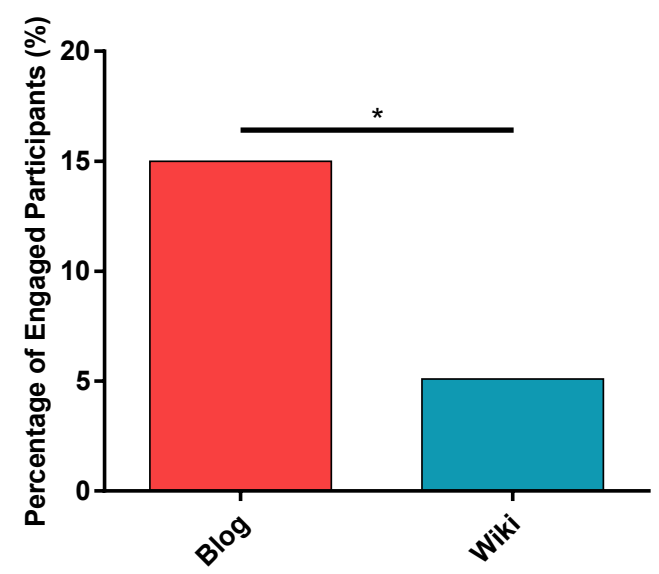

Figure 2. The percentage of participants who signed up for the blog recommendation list and/or blog subscription was significantly higher for participants who read the blog article compared to those who read the Wikipedia article. ${ }^{*} p<0.05$ 
This group difference supports the hypothesis that the Sustainable Nano blog is achieving its goal of promoting engagement in readers, and provides preliminary evidence that blogs have the potential to be effective at science communication goals in ways that are qualitatively different from equally accessible online expository sources like Wikipedia.

As predicted, the group difference in engagement differs from the results for knowledge and attitude; there was little change in these measures from before the readings to after, when participants were allowed to view their prior ratings and change them if desired. Participants on average shifted their responses only 0.028 for the blog group and 0.044 for the Wiki group on a 5 -point scale for the knowledge questions ( 1 =strongly true, $5=$ strongly false), with a positive shift indicating increased understanding. For the attitude questions, the average shift in score was 0.002 for both groups on a 5 -point scale ( 1 =strongly agree, $5=$ strongly disagree), where a positive change indicates an increase in positive attitude toward science. Not only were these changes small in magnitude; neither was statistically significant. The content of the blog post and Wikipedia article in the study did not explicitly relate to the questions in the nanotechnology knowledge quiz, nor advocate for any particular attitude toward science and technology, and therefore we did not expect readers to change in those measures. It is worth emphasizing the distinction between knowledge and attitude measures and engagement in the current study, because to our knowledge this is the first example of such a demonstration. Different results might be expected if the knowledge quiz questions related to the specific information in the readings; indeed, it would be interesting to follow up with a study using a blog post whose content was directly relevant to the nanotechnology quiz items, and with a control (Wikipedia) text that was more closely aligned to the topic of the blog post, in order to study whether readers' information retention might be better with a blog post designed for audience engagement as opposed to the purely expository style of Wikipedia (or even a textbook excerpt). Following up on Sheufele and Lewenstein's discussion of "low-information rationality,"(Scheufele \& Lewenstein, 2005) it could be particularly interesting to select a blog post for study that has high traffic based on organic search (in other words, a post that readers frequently find based on a specific Google inquiry), to see whether readers who come to the blog to learn a specific piece of information might then be inspired to engage more than they originally intended.

\section{Demographic Variables}

We examined several demographic variables to explore whether they interacted with group assignment to affect engagement outcome. Partly due to small sample sizes in each category, we did not find any significant differences. Nevertheless, we observed some interesting qualitative patterns that may warrant further investigation.

When comparing men and women participants in the study, there appeared to be an interesting qualitative trend that women were more engaged than men (14\% vs $9 \%)$, although gender and engagement were not statistically dependent, $x_{2}(1, N=130)=0.033, p=0.86$. Prior research has found that women are more likely than men to follow a brand on social media (The Nielsen Company, 2012) which could suggest that they would be more likely to subscribe to a blog site. 
This question merits further study, as gender differences in "engagement" behavior could inform how researchers design future research on this topic.

Interesting qualitative patterns also emerged for age. Although reading the blog seemed to inspire engagement for participants of all ages, and no statistically significant group differences emerged, qualitative examination suggests that the only participants in the Wikipedia group who demonstrated engagement were 45 years old and older (Figure 3). Further follow-up would be needed to verify whether this trend reflected a genuine age effect. The difference was not statistically significant partly because there were so few people in the younger groups - the overall engagement demonstrated in the Wikipedia group is such a small proportion $(5.1 \%)$ that an apparent difference among groups (some containing fewer than 20 participants) is difficult to interpret meaningfully.

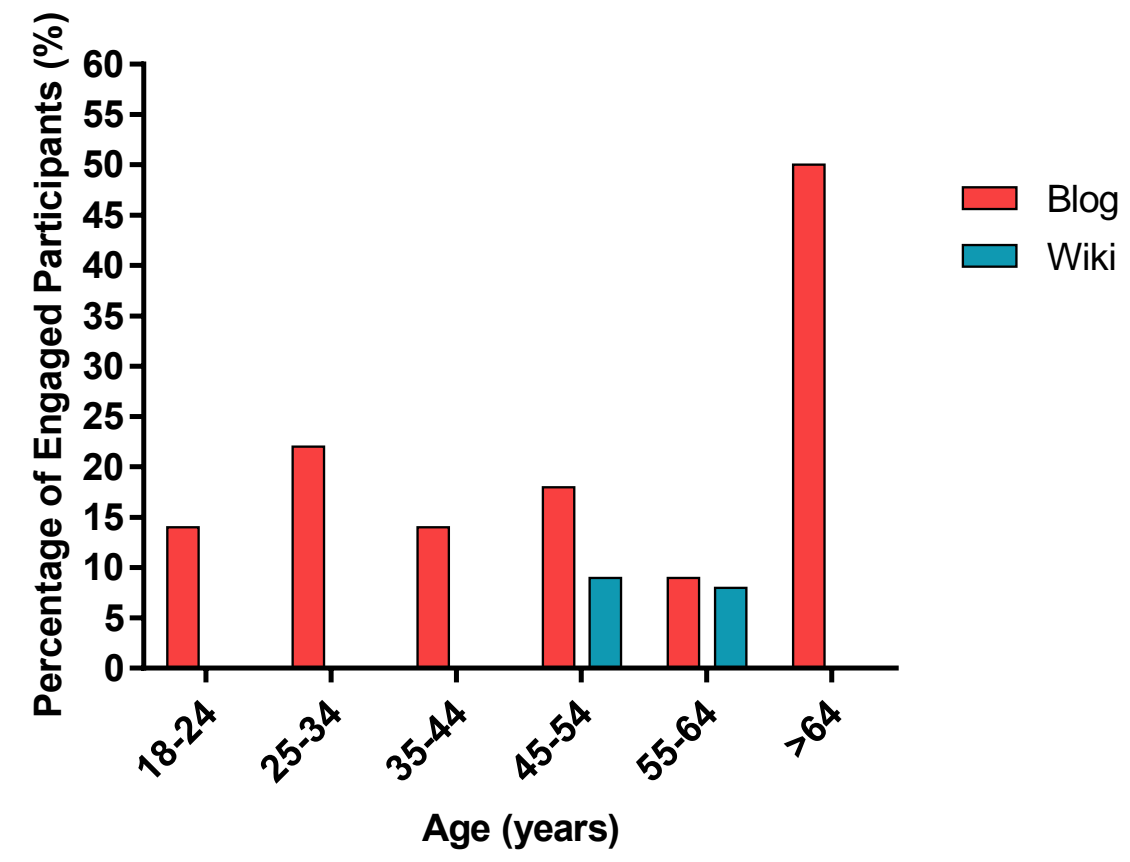

Figure 3. The percentage of participants in different age groups that were engaged based on which article they were assigned.

Regarding education, more participants in the study had somewhat higher education than would be expected in the general population of the state or of State Fair attendees $(79 \%$ with higher education compared to $35 \%$ for the state (Minnesota Office of Higher Education, 2017) and 37.5\% for the State Fair (Minnesota State Fair \& Nielsen Scarborough, 2018)). This may reflect the selfselection of participants who approach the Driven to Discover building, and/or a general characteristic of blogs as an outreach tool, if those with higher education are more likely to seek out this format. (Moreover, the Driven to Discover building is branded in relationship with the University of Minnesota, which might potentially pose a visual/cultural barrier to some potential participants.) These issues pose interesting questions to pursue in future studies. Unfortunately, 
there were not enough participants in each educational category of our study to assess whether the blog was more effective at engaging some groups than others.

One advantage of the State Fair as a research venue is that it draws attendees from all over the state, allowing for participant recruitment across a wider range of geographic areas than is ordinarily possible with studies on campus. We had $74 \%$ urban participants vs $26 \%$ non-urban participants, and we saw no overall difference in engagement depending on the geographic origin of the participants. However, an interesting trend emerged showing that the urban participants were more engaged after reading the blog post compared to the Wikipedia article $\left(x^{2}(1, N=89)=\right.$ $5.6706, p=0.01725)$, whereas participants from non-urban areas were equally engaged after reading the blog or Wikipedia article $\left(x_{2}(1, N=30)=0.10819, p=0.7422\right)$. While the small sample size here limits the generalizability of this phenomenon, this trend merits further investigation to determine if blog posts are more effective at engaging an urban-based audience than traditional expository-styled texts.

Finally, no significant differences were observed based on race or ethnicity, but unfortunately our sample was very skewed toward white, non-Hispanic/Latino participants and so it would have been difficult to observe any differences in our sample. The population of Minnesota overall is approximately $20 \%$ people of color (United States Census Bureau, 2017), and we have not been able to find any information on the proportion for those attending the State Fair. Further information on demographics is available in the Supplemental Information.

\section{Limitations and future directions}

Perhaps the most obvious weakness in the current study is the relatively small number and limited diversity of participants. Although collecting data at the State Fair provided a far broader study population than a typical convenience group on a university campus, the demographics were still not representative of the local population, particularly with regards to race and education. Further study is needed to delve into whether these factors would affect the observed engagement effect; moreover, the self-selection at play in our study participation is likely relevant to the question of who chooses to read science blogs in general. In addition to the demographic factors already discussed, there is a need for more research into who reads science blogs and why, but our findings suggest that at least for some individuals, reading one blog post may lead to further engagement with similar material.

Due to the exploratory nature of the study and our decision to exclude the "pre-engaged" subgroup, the statistical power in the dataset was insufficient to quantitatively evaluate some potentially interesting demographic patterns that emerged. For example, the only participants in the Wiki group who demonstrated engagement were 45 years old and older, and women overall demonstrated greater engagement than men. Neither of these trends were statistically significant, but would be good candidates for further investigation.

Another limitation in the study arose due to a software error in our survey platform, which resulted in the loss of all responses to the question, "How often do you read blogs about scientific issues or emerging technologies?" The loss of this anchor data point hinders our interpretation of the 
relationships among our other measures of engagement. As described above, we were initially surprised to find that people who chose to read the second "article" were significantly more likely to sign up for the other two "engagement" measures (signing up for the Sustainable Nano email updates and the recommended blog list), regardless of their random group assignment. Our working assumption (in the absence of the blog readership question data) is that those who read the second article were demonstrating that they came to the study "pre-engaged," regardless of group assignment. An alternate explanation is that the optional second article (a blog post entitled, "What do gold nanoparticles have to do with wrinkly skin?") may have been so compelling that it uniquely encouraged engagement, overwhelming the effect of blog vs. Wiki group assignment. It would be helpful for a future study to have multiple versions of both blog posts and Wikipedia articles to confirm the blog vs. Wiki difference independent of individual post content.

Further exploring the effect (or lack thereof) of individual post content in the "control" article would also be useful for examining the question of reader engagement. In the current study, we controlled for length (approximate word count) and number of images, but other aspects of the readings could be manipulated in order to understand how they relate to the likelihood of readers' interest in pursuing further interactions with science blogs. Rather than having two readings generally about butterflies, we could more closely match the content in order to compare the effects of the blog's pseudo-narrative writing style with Wikipedia's expository style. Existing literature on learning through reading suggests that expository styles can be more challenging for readers to comprehend than narrative styles (McCormick, 2007), especially for those who do not already have a strong background about the material (Hall, 2004).

\section{Conclusions}

This study provides some of the first experimental evidence that reading a science blog can promote reader engagement. Specifically, participants randomly assigned to read a Sustainable Nano blog post were more likely to volunteer to receive further email updates than those assigned to read a Wikipedia article of similar length. Many important questions remain regarding the effectiveness of science blogs at achieving science communication goals, including not only different goals and characteristics of blogs themselves but also many reader variables.

\section{Supplementary Information}

Survey readings and optional reading; engagement questions; demographic information for all participants; geographic maps of participant zip codes

\section{References}

American Geophysical Union. (2019). AGU Blogosphere. Retrieved from https://blogs.agu.org/ Boholm, A., \& Larsson, S. (2019). What is the problem? A literature review on challenges facing the communication of nanotechnology to the public. Journal of Nanoparticle Research, $21,86-86$.

Center for Sustainable Nanotechnology. (2019). Sustainable Nano. Retrieved from http://sustainable-nano.com

Chemistry Blog. (2019). Chemistry Blog. Retrieved from http://www.chemistry-blog.com 
Collins, K., Shiffman, D., \& Rock, J. (2016). How Are Scientists Using Social Media in the Workplace? PLoS ONE, 11(10), e0162680-e0162680.

doi:10.1371/journal.pone.0162680

Doğangün, M. (2015). Mimicking Nature's Nanotechnology: From a Butterfly Wing to AntiCounterfeit Technologies. Retrieved from http://sustainablenano.com/2015/01/20/mimicking-natures-nanotechnology/

Francl-Donnay, M. (2019). The Culture of Chemistry. Retrieved from http://cultureofchemistry.fieldofscience.com/

Fraser, B. J. (1981). Test of science-related attitudes (TOSRA) handbook. Hawthorn, Victoria: Australian Council for Educational Research.

Hall, L. A. (2004). Comprehending expository text: Promising strategies for struggling readers and students with reading disabilities? Reading Research and Instruction, 44, 75-95. doi:10.1080/19388070409558427

Hoang, J. K., McCall, J., Dixon, A. F., Fitzgerald, R. T., \& Gaillard, F. (2015). Using social media to share your radiology research: how effective is a blog post? Journal of the Americal College of Radiology, 12, 760-765.

Jarreau, P. B. (2015). Science bloggers' self-perceived communication roles. Journal of Science Communication, 14, A02-A02.

Jarreau, P. B., \& Porter, L. (2018). Science in the Social Media Age: Profiles of Science Blog Readers. Journalism and Mass Communication Quarterly, 95, 142-168. doi: $10.1177 / 1077699016685558$

Kouper, I. (2010). Science blogs and public engagement with science: Practices, challenges, and opportunities. Journal of Science Communication, 9(1), A02.

Lewenstein, B. (2016). Public Engagement. Views. Retrieved from https://www.informalscience.org/news-views/public-engagement

McCormick, S. (2007). Instructing students who have literacy problems (5th ed.). New Jersey: Pearson Merrill Prentice Hall.

McGeachy, A. (2014). What do gold nanoparticles have to do with wrinkly skin? Retrieved from http://sustainable-nano.com/2014/11/25/gold-in-cosmetics/

McGlynn, T. (2019). Small Pond Science. Retrieved from http://smallpondscience.com

Minnesota Office of Higher Education. (2017). Educational Attainment: How educated are Minnesota's adult population (age 25 to 64 )? Retrieved from http://www.ohe.state.mn.us/fc/2108/pg.cfm

Minnesota State Fair, \& Nielsen Scarborough. (2018). Minnesota State Fair Guest Demographic Sheet. Retrieved from https://assets.mnstatefair.org/pdf/19-Demo-Sheet.pdf

Natelson, D. (2019). Nanoscale Views. Retrieved from http://nanoscale.blogspot.com/

Nature. (2019). Nature.com Blogs. Retrieved from http://blogs.nature.com/

Plait, P. (2019). Bad Astronomy. Retrieved from https://www.syfy.com/tags/bad-astronomy

Scheufele, D. A., \& Lewenstein, B. V. (2005). The public and nanotechnology: How citizens make sense of emerging technologies. Journal of Nanoparticle Research, 7(6), 659-667.

ScienceGrrl. (2019). ScienceGrrl. Retrieved from https://sciencegrrl.co.uk/

Scientific American. (2019). Scientific American Blogs. Retrieved from https://blogs.scientificamerican.com/

Scientopia. (2019). Scientopia: a science blogging collective. Retrieved from http://scientopia.org

Su, L. Y.-F., Akin, H., Brossard, D., Scheufele, D. A., \& Xenos, M. A. (2015). Science News Consumption Patterns and Their Implications for Public Understanding of Science. Journalism \& Mass Communication Quarterly, 92(3), 597-616. doi:10.1177/1077699015586415 
Su, L. Y.-F., Cacciatore, M. A., Scheufele, D. A., Brossard, D., \& Xenos, M. A. (2014). Inequalities in Scientific Understanding: Differentiating Between Factual and Perceived Knowledge Gaps. Science Communication, 36(3), 352-378.

United States Census Bureau. (2017). State Population by Characteristics: 2010-2017. Retrieved from

Wikipedia. (2017). Butterfly. Retrieved from https://en.wikipedia.org/wiki/Butterfly

Zivkovic, B. (2012). Science Blogs - Definition, and a History. Retrieved from https://blogs.scientificamerican.com/a-blog-around-the-clock/science-blogs-definitionand-a-history/ 